\title{
Hypothesis Verification for Designing Flyback Booster by Analysis of Variance Visualized on Triangular Matrix Representations
}

\author{
Kazuhisa Chiba $^{1(\bowtie)}$, Taiki Hatta ${ }^{1}$, and Masahiro Kanazaki ${ }^{2}$ \\ 1 The University of Electro-Communications, \\ 1-5-1, Chofugaoka, Chofu, Tokyo 182-8585, Japan \\ kazchiba@uec.ac.jp \\ 2 Tokyo Metropolitan University, \\ 6-6, Asahigaoka, Hino, Tokyo 191-0065, Japan \\ kana@tmu.ac.jp \\ http://www.di.mi.uec.ac.jp/chiba/index_e.html
}

\begin{abstract}
This study performed data mining for nondominatedsolution datasets of flyback-booster geometry for next-generation space transportation procured by evolutionary computation. We prepared two datasets of nondominated solutions due to two problem definitions, which differ merely in the definition of some design variables based on a design hypothesis gained from evolutionary-computation results. This study aims at verifying the hypothesis by applying mining to these two datasets to elucidate the contrast in the influence of the design variables. We used functional analysis of variance for data mining; scrutinized the effects of single and two-combined design variables. Furthermore, intuitive visualization by triangular matrix representations could distinguish the discrepancy between the obtained results. The consequence has verified the significance of the hypothesis; it revealed that the discontinuous surface naturally evaded in the hypersonic range because of surface temperature upsurge is capable of enhancing the lift-to-drag ratio in the low-speed range; the hypothesis grew into a new design problem.
\end{abstract}

Keywords: Design informatics $\cdot$ Space transportation $\cdot$ Information visualization $\cdot$ Real-world design problems

\section{Introduction}

High-frequency and price reduction of space transportation are urgent priorities by the recent gains in the demands of small satellites. Under this circumstance, reusable launch systems (RLSs) are one of the realizability in several aspects to attain the purposes as a successor of the Space Shuttle. Hence, studies have

Supported by JHPCN, JSPS KAKENHI, and IFS Tohoku University.

(C) Springer Nature Singapore Pte Ltd. 2020

Y. Tan et al. (Eds.): DMBD 2020, CCIS 1234, pp. 34-44, 2020.

https://doi.org/10.1007/978-981-15-7205-0_4 
lately activated again for developing reusable space transportation, mainly in China [16], Russia [11], the E.U. [14], and the U.S [7]. Meanwhile, Japan is currently conducting a collaborative research project on two-stage RLS among some universities. Launch experiments of a prototype model are underway to establish a control law for launching an authentic vehicle [6]. The project is designing trajectories and body geometries at the same time; namely, we are studying the conceptual design for an RLS.

The 1st evolutionary optimization for 3D geometry design accidentally created a discontinuous ditch on the back of flyback boosters [15]. The hypersonic region covered in the trajectory generally eludes the discontinuity of body surface that causes the temperature to rise due to adiabatic compression. Therefore, the geometry representation unit in the optimal design system solved the inverse problem and modified the discontinuous ditch intentionally to make them continuous. When we evolved the 2nd optimization in the equal generation number using the identical initial population as the 1st optimization, the lift-to-drag ratio $(L / D)$ in the transonic/supersonic range declined wholly [8]. This result implies the hypothesis for the body design that achieves moderate accomplishments while improving the aerodynamic performance in the low-speed region by the deliberate discontinuity of the body surface promoting the vortex lift.

This study aims to verify the design hypothesis by performing data mining on nondominated-solution sets of flyback-booster geometry procured by evolutionary computations (ECs) and elucidating the contribution of design variables to the $L / D$, namely the study is an application of data mining to real-world design. The mining target is two sets of nondominated solutions gained for two problems that vary simply in the definition of some design variables based on the design hypothesis. We scrutinize distinction in the influence of design variables on the $L / D$. Since the difference in the optimization results revealed an apparent decrease in the $L / D$, it infers that the character of surface ditches would emerge not to be small; the data mining will utilize a functional analysis of variance to examine each design variable and combinations between two design variables thoroughly. Furthermore, visualization by triangular matrix representations (TMR) [1] will facilitate intuitive perceptions of the procured distinctions.

We structure this article as follows. Section 2 explains the problem settings and outlines the ways of data mining concisely. Section 3 reviews the design information caused by the optimization. In Sect. 4, we assess the gained results by data mining. Section 5 concludes this article.

\section{Problem Settings}

At present, we are promoting research on space transportation systems at several domestic universities, including Kyushu Institute of Technology, which designs and develops fully reusable space transportation WIRES [6]. We tried to design space transportation, commencing from the flight path optimized with WIRES. Initially, although the optimum trajectory also alters according to the change of the geometry, the hurdle for generating the aerodynamic performance matrix necessary for trajectory optimization is high, so it is a future work. 
Table 1. Definition of design variables.

\begin{tabular}{l|l|l}
\hline Section number & Design-variable S/N number & Symbol (refer to Fig. 2) \\
\hline \multirow{2}{*}{$(2)$} & $1,3,5,7,9$ & $V_{y 1}, V_{y 2}, V_{y 3}, V_{y 4}, V_{y 5}$ \\
\cline { 2 - 3 } & $2,4,6,8,10$ & $V_{z 1}, V_{z 2}, V_{z 3}, V_{z 4}, V_{z 5}$ \\
\hline \multirow{3}{*}{4} & $11,13,15,17,19$ & $V_{y 1}, V_{y 2}, V_{y 3}, V_{y 4}, V_{y 5}$ \\
\cline { 2 - 3 } & 12 & $V_{z 1}$ \\
\cline { 2 - 3 } & $14,16,18$ & $V_{z 2}, V_{z 3}, V_{z 4}$ \\
\cline { 2 - 3 } & 20 & $V_{z 5}$ \\
\hline \multirow{5}{*}{5} & $21,23,25,27,29$ & $V_{y 1}, V_{y 2}, V_{y 3}, V_{y 4}, V_{y 5}$ \\
\cline { 2 - 3 } & 22 & $V_{z 1}$ \\
\cline { 2 - 3 } & $24,26,28$, & $V_{z 2}, V_{z 3}, V_{z 4}$ \\
\cline { 2 - 3 } & 30 & $V_{y 1}, V_{y 2}, V_{y 3}, V_{y 4}, V_{y 5}$ \\
\hline & $31,33,35,37,39$ & $V_{z 1}$ \\
\cline { 2 - 3 } & 32, & $V_{z 2}, V_{z 3}, V_{z 4}$ \\
\cline { 2 - 3 } & $34,36,38$, & \\
\cline { 2 - 3 } & 40 & \\
\hline
\end{tabular}

The trajectory used in this study assumes an injection of $10 \mathrm{t}$ payload into the orbit from the Tanegashima Space Center and a circular orbit at an altitude of $350 \mathrm{~km}$. Based on this, they were defining the aerodynamic performance optimization in three points of transonic, supersonic, and hypersonic speeds. At the defined transonic/supersonic design point, the booster is the point where we have to obtain a range for fly back to the launch field. At the hypersonic design point where a booster and an orbiter separate, at this point, heave the altitude and take a sequence to secure the range margin.

\subsection{Optimization Problem}

This problem defines six objective functions: three for aerodynamics (maximizing the $L / D$ at $M=0.65,2.3$, and 6.8), one for the structures (minimizing empty weight), and two for aerodynamic heating (minimizing body surface temperature and body surface area where thermal protection systems attaches).

As shown in Fig. 1, we generate six cross-sectional shapes for the $x$ axis direction. We utilize section numbers (1) and (6) only to satisfy later-described constraint conditions, and the cross-sectional shape change in optimization is four cross-sections with 2 to 5 . Since one cross-section uses ten design variables as shown in Fig. 2, the total of design variables is 40. Table 1 explains the definition of each design variable. 


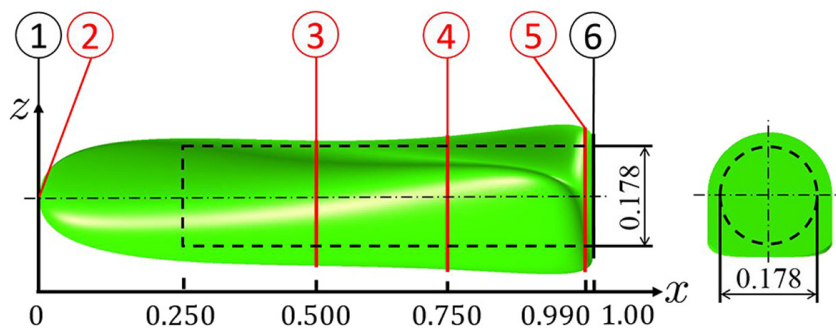

Fig. 1. Left: cross-section positions, right: tail surface. The dotted line describes a fuel tank to be a fixed size.

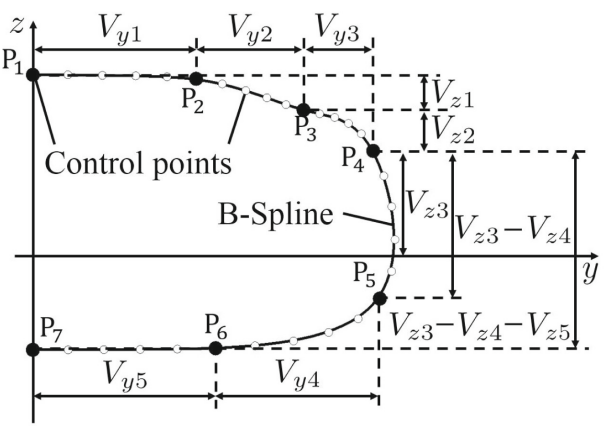

(a) cross-section (2)

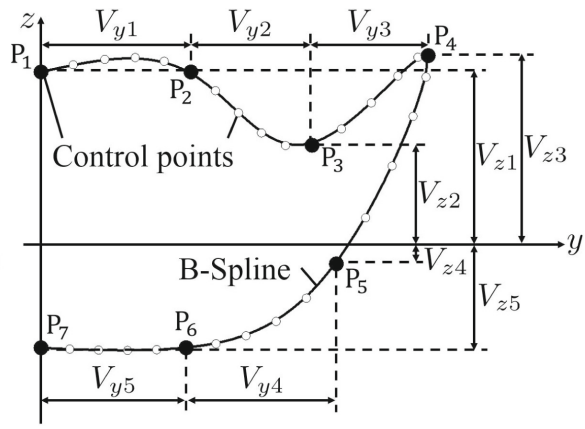

(b) cross-sections (3) to (5)

Fig. 2. Relations among design variables $V_{n}$ and control points $\mathrm{P}_{m}$ at each crosssection. White points from $\mathrm{P}_{i}$ to $\mathrm{P}_{i+1}$ are the subordinate control points of $\mathrm{P}_{m}$.

We define constraints on the geometry; no constraint provides for the objective functions. When making individuals satisfying the geometrical constraints for the population size, the optimization system ceases creating ten individuals for one generation.

\subsection{Optimizer}

Since one of the information desired by the multiobjective optimization is the executable structure of the objective-function space, we employed an EC that performs a global search for the optimization method; we made a fully automated design system [2]. This study adopted the strength Pareto evolutionary algorithm 2 (SPEA2) [17] in many ECs due to conventionality. We respectively chose simulated binary crossover [4] and polynomial mutation [5] in the standard.

\subsection{Data-Mining Technique}

A functional analysis of variance (ANOVA) quantifies the contribution rates to the variance of a model [9]. ANOVA decomposes the total variance of the model 
into that of each design variable and their interactions to estimate their impact quantitatively by integrating values out of the model $\widehat{y}$. The Kriging model [10] for an objective value $y(\boldsymbol{x})$ of design variables $\boldsymbol{x}(\operatorname{dim} \boldsymbol{x}=N)$

$$
y(\boldsymbol{x})=\beta+Z(\boldsymbol{x})
$$

predicts the model $\widehat{y} . \beta$ and $Z(\boldsymbol{x})$ are a constant term of the model and a Gaussian random process with zero mean and variance of $\sigma^{2}$, respectively. In this case, the single effect of $x_{i}$ is

$$
\begin{aligned}
\widehat{\mu}\left(x_{i}\right)= & -\underbrace{\int_{D^{N}} \widehat{y}(\boldsymbol{x}) \mathrm{d} x_{1} \mathrm{~d} x_{2} \cdots \mathrm{d} x_{N}}_{\widehat{\mu}(\boldsymbol{x})} \\
& +\int_{D^{N-1}} \widehat{y}(\boldsymbol{x}) \mathrm{d} x_{1} \mathrm{~d} x_{2} \cdots \mathrm{d} x_{i-1} \mathrm{~d} x_{i+1} \cdots \mathrm{d} x_{N}
\end{aligned}
$$

and likewise the combined impact of $x_{i}$ and $x_{j}(i<j)$ is

$$
\begin{aligned}
\widehat{\mu}\left(x_{i, j}\right)= & -\widehat{\mu}(\boldsymbol{x})-\widehat{\mu}\left(x_{i}\right)-\widehat{\mu}\left(x_{j}\right) \\
& +\int_{D^{N-2}} \widehat{y}(\boldsymbol{x}) \mathrm{d} x_{1} \mathrm{~d} x_{2} \cdots \mathrm{d} x_{i-1} \mathrm{~d} x_{i+1} \cdots \mathrm{d} x_{j-1} \mathrm{~d} x_{j+1} \cdots \mathrm{d} x_{N} .
\end{aligned}
$$

The variance $\widehat{\varepsilon}\left(x_{i}\right)$ caused by $x_{i}$ is

$$
\widehat{\varepsilon}\left(x_{i}\right)=\int\left(\widehat{\mu}\left(x_{i}\right)\right)^{2} \mathrm{~d} x_{i},
$$

SO

$$
p\left(x_{i}\right)=\frac{\widehat{\varepsilon}\left(x_{i}\right)}{\widehat{\varepsilon}(\boldsymbol{x})}
$$

distributes the proportion on the contribution of $x_{i}$.

Various information visualization techniques express database search results as sturdy devices for knowledge discovery [12]. The filtering techniques, including TMR [1], have proven to be serviceable for tasks where visual representations can find relationships, clusters, outliers, gaps, and other patterns [13].

\section{Review of the Optimization Results}

This section concisely reviews the knowledge that the optimizations have already indicated, which is crucial for interpreting the data-mining results.

\subsection{Knowledge from the Optimization Results}

The ditch brings a positive effect in the lift; it causes a negative influence on the drag. As a result of the optimization, we anticipate that the ditch will have a positive impact on the low-speed range $L / D$. Therefore, this study focuses on improving the low-speed range $L / D$ and does not discuss the result at $M=6.8$. 
Difference of Design Strategies. Optimal geometries of each objective function for the $L / D$ explicates the following diversity in design strategies.

- $L / D$ at $M=0.65$ grows by raising the lift.

- $L / D$ at $M=6.8$ rises by reducing the drag.

The optimization makes the body slender to reduce the drag in the hypersonic range. Since slim bodies concomitantly enable to decrease the surface temperature, they simultaneously improve three of the six objective functions; it must stipulate the evolutionary trend.

- Strategy for increasing the $L / D$ at $M=2.3$ is related to that for the $L / D$ at $M=6.8$, but with scope for a rising lift.

\section{Influence of Ditch}

- Positive effect in the lift: ditches facilitate vortex form; they grow vortex lift.

- Negative influence on the drag: the induced drag rises due to the vortex lift; the friction drag first increases with the expansion of the wetted area.

\subsection{Design Information from SPM}

The 1st and 2nd optimization results shown in Fig. 3 present the effect of the presence or absence of a ditch on the transonic/supersonic $L / D$ as follows.

- To eliminate ditch deteriorates the $L / D$ at $M=0.65$, approximately $5.4 \%$.

- To eliminate ditch deteriorates the $L / D$ at $M=2.3$, approximately $1.9 \%$.

- The $L / D$ at $M=6.8$ also declines, but it links to the $L / D$ at $M=2.3$.

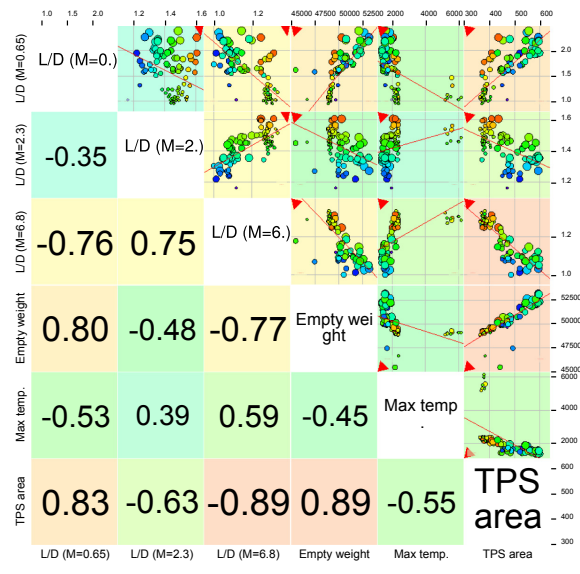

(a) 1st result considering ditch.

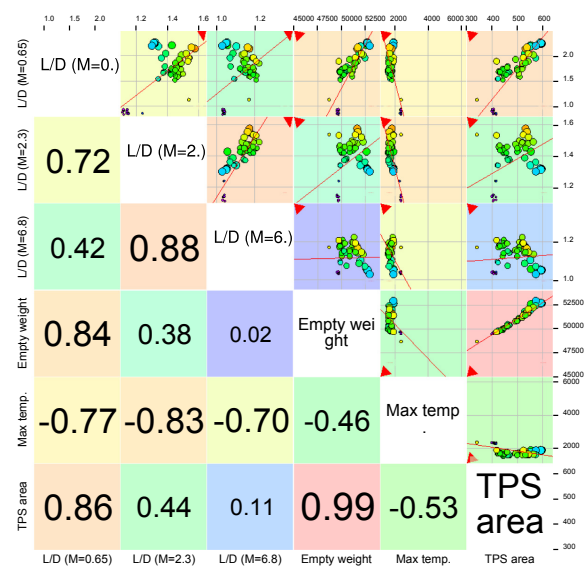

(b) 2nd result without considering ditch.

Fig. 3. Scatter plot matrix of nondominated solutions for 30 generations by SPEA2 in the objective-function space. Red lines/triangles signify regression lines/optimum directions. Numbers on lower triangular matrices specify correlation coefficients. (Color figure online) 
Originally, $M=0.65$ is more reliant on the lift than $M=2.3$ for improving the $L / D$, so we infer that to add ditch produces a more positive effect on the lift than a negative impact on the drag.

\section{Results by Data Mining}

The purpose of data mining is to verify whether the accidentally created ditches on the back of boosters contribute to improving $L / D$. So, we have to prove the following two perspectives: (1) the dataset of 1st optimization result that considers ditches affects design variables involved in ditch generation, (2) the dataset of 2nd optimization result that excludes ditches does not impact design variables required for ditch representation. Note that the results of ANOVA gained for each objective function quantify the contribution of each design variable, assuming that the total influence on an objective function is $100 \%$. We cannot quantitatively discuss the relative effects among objective functions.

\subsection{Result for Dataset with Ditch}

$\boldsymbol{L} / \boldsymbol{D}$ at $\boldsymbol{M}=\mathbf{0 . 6 5}$. TMR shown in Fig. 4(a) indicates that the design variables with substantial influence are $d v 34, d v 37$, and $d v 40$ as well as with weak effect are $d v 12, d v 20, d v 22$, and $d v 27$. The beneficial design variables register that the design strategies for improving $L / D$ at $M=0.65$ are (a) spreading the body in the spanwise direction and making the bottom as flat as possible, and (b) providing ditches. To prolong the wetted area, that is, to stretch the body in the spanwise direction is the most valuable for raising the lift, which effects on improving $L / D$ at $M=0.65$. Therefore, the influence of the design variables ( $d v 20, d v 27, d v 37, d v 40)$ for expanding the fuselage in the spanwise direction and flattening the bottom surface is relatively more massive than the design variables $(d v 12, d v 22, d v 34)$ for providing ditches. However, we can affirm that the provision of the ditch is undoubtedly useful in improving the $L / D$.

1. $d v 34\left(V_{z 2}^{(5)}\right)$ : to raise the difference from $V_{z 1}$ boosts the curvature of the B-Spline curve from $\mathrm{P}_{1}$ to $\mathrm{P}_{3}$, resulting in a deeper ditch; the ditch affects the low-speed $L / D$. Note that $V_{z 1}$ tends to be large to serve the constraint of securing the tank volume, so $V_{z 2}$ inclines to be small.

2. $d v 37\left(V_{y 4}^{5}\right)$ : decreasing this value widens the flat bottom region in the span direction; growing the pressure on the bottom of the fuselage gains the lift.

3. $d v 40\left(V_{z 5}^{5}\right)$ : by reducing the bottom thickness and flattening fuselage in the z-direction, the base matures a flat geometry. The identical design concept as $d v 37$ contributes to lift repair.

\footnotetext{
${ }^{1}$ For example, $d v 34$ symbolizes the design variable $V_{z 2}$ of the fifth cross-section displayed in Table 1 and Fig. 2, so this paper expresses it as $V_{z 2}^{5}$.
} 
$\boldsymbol{L} / \boldsymbol{D}$ at $\boldsymbol{M}=\mathbf{2 . 3}$. TMR shown in Fig. 4(b) indicates that the design variables with substantial influence are $d v 14, d v 17, d v 22$, and $d v 40$. Since drag alleviation helps improve $L / D$ at $M=2.3$, unlike optimizing the $L / D$ at $M=0.65$, no geometry widens horizontally and tends to be elongated. Hence, the impact of the design variables $(d v 17, d v 40)$ that expand the body horizontally and the design variables $(d v 14, d v 22)$ that supplement ditches to the lift is relatively robust in the latter.

\subsection{Result for Dataset Without Ditch}

$\boldsymbol{L} / \boldsymbol{D}$ at $\boldsymbol{M}=\mathbf{0 . 6 5}$. Since all design variables indicated by TMR shown in Fig. 5(a) are irrelevant to ditches, ditches do not affect on the $L / D$. The abilities of design variables that have direct and indirect effects are abstracted below.

- design variables with direct effects

1. $d v 10: V_{z 5}^{2}$ has the effect of gaining the lift by making the bottom of the body nose as flat as possible.

2. $d v 35: V_{y 3}^{5}$ extends the area near the body tail in the spanwise direction and creates a stabilizer to enhance the lift.

- design variables with indirect effects

1. $d v 11, d v 23: V_{y 1}^{3}$ and $V_{y 2}^{(4)}$ widen the body sideways and gain the lift.

2. $d v 40: V_{z 5}^{5}$ raises the lift by flattening the bottom near the body tail.

$\boldsymbol{L} / \boldsymbol{D}$ at $\boldsymbol{M}=\mathbf{2 . 3}$. All design variables indicated by TMR shown in Fig. 5(b) are independent of the ditch, so the impact of the ditch faded away in the $L / D$ at $M=2.3$ as well as in the $L / D$ at $M=0.65$. The capabilities of design variables that affect the $L / D$ at $M=2.3$, as shown by TMR, are as follows.

- design variables with direct influences

1. $d v 10: V_{z 5}^{2}$ is a variable that ultimately transforms the $L / D$ because it is useful in raising the lift by flattening the bottom of the body's nose and in diminishing the drag by building the curvature.

- design variables with indirect effects

1. $d v 8, d v 20, d v 40: V_{z 4}^{2}, V_{z 5}^{3}$, and $V_{z 5}^{5}$ have effects comparable to $d v 10$.

2. $d v 22\left(V_{z 1}^{(4)}\right)$ : the direct effect is weak, and the result is more robust when combined with $d v 10$, which implies that the cross-sectional shape transformation in the flow direction affects the $L / D$. Generally, in the supersonic range, the cross-sectional area distribution of the body in the flow direction desires alteration monotonically from the sonic boom theory [3]. The combination of $d v 10$ and $d v 22$ must deviate from a monotonic increase, leading to a rise in the wave drag, resulting in the $L / D$ deterioration. In particular, since $d v 22$ represents the cross-section (4), its cross-section is likely to disturb area distribution because there commences forming a stabilizer. In any case, $d v 22$ does not affect the lift. 


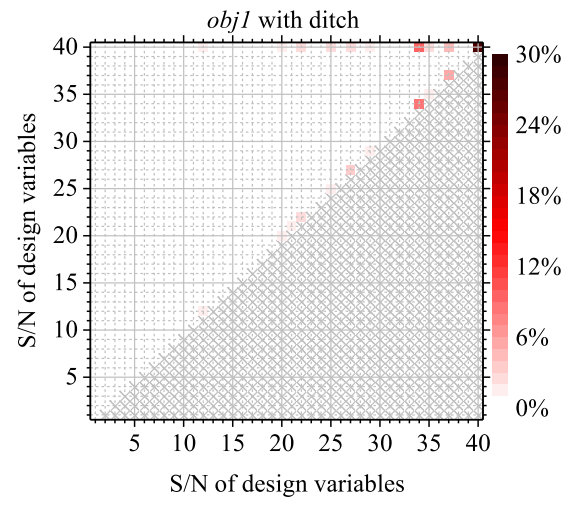

(a)

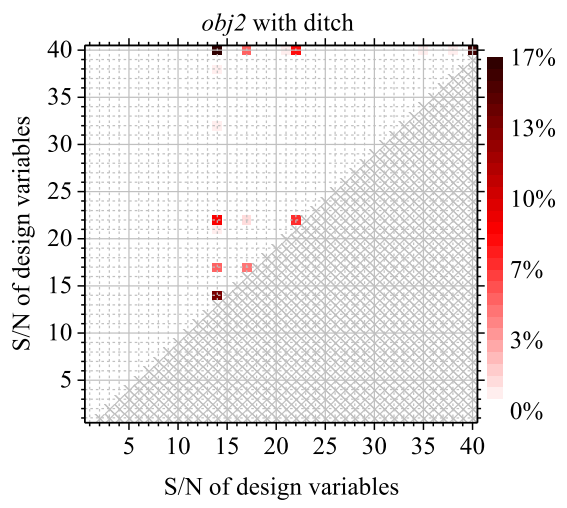

(b)

Fig. 4. TMR of ANOVA results for the dataset of the 1st optimization, which accidentally deals with ditches. (a) $L / D$ at $M=0.65$ and (b) $L / D$ at $M=2.3$.

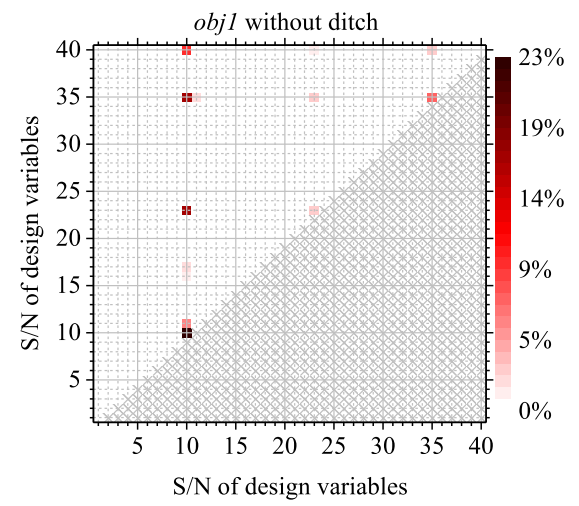

(a)

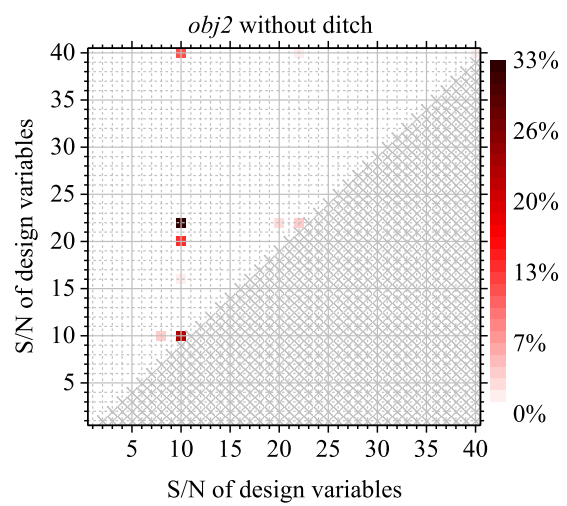

(b)

Fig. 5. TMR of ANOVA results for the dataset of the 2nd optimization, which solves the inverse problem to eliminate ditches. (a) $L / D$ at $M=0.65$ and (b) $L / D$ at $M=2.3$.

\section{Conclusion}

This research performed data mining by functional analysis of variance on multidisciplinary many-objective optimization results for a real-world problem and intuitively visualized the mining results using triangular matrix representations. We aimed at verifying a design hypothesis for a flyback booster in space transportation. Ditches avert surface temperature surge caused by adiabatic compression in the hypersonic range. However, they on the body back facilitated the lift rise in the transonic and supersonic ranges. We proved that ditches contributed to the increment in the low-speed lift-to-drag ratio; the study has affirmed that 
the hypothesis was correct. We have consequently gained a new design problem that examines how to add ditches to the body surface.

\section{References}

1. Birkenmeier, G.F., Heatherly, H.E., Kim, J.Y., Park, J.K.: Triangular matrix representations. J. Algebra 230(2), 558-595 (2000). https://doi.org/10.1006/jabr.2000. 8328

2. Chiba, K., Sumimoto, T., Sawahara, M.: Completely automated system for evolutionary design optimization with unstructured computational fluid dynamics. In: Proceedings of International Conference on Intelligent Systems, Metaheuristics \& Swarm Intelligence. ACM (2019). https://doi.org/10.1145/3325773.3325778

3. Darden, C.M.: Sonic boom theory - its status in prediction and minimization. J. Aircr. 14, 569-576 (1977)

4. Deb, K., Agrawal, R.B.: Simulated binary crossover for continuous search space. Complex Syst. 9(2), 115-148 (1995)

5. Deb, K., Agrawal, R.B.: A combined genetic adaptive search (GeneAS) for engineering design. Comput. Sci. Inform. 26, 30-45 (1996)

6. Fujikawa, T., et al.: Research and development of winged reusable rocket: current status of experimental vehicles and future plans. In: Proceedings on AsiaPacific International Symposium on Aerospace Technology. JSASS, Soul, Republic of Korea (2017)

7. Harris, M.: The heavy lift: blue origin's next rocket engine could power our return to the moon. IEEE Spectr. 56, 26-30 (2019). https://doi.org/10.1109/MSPEC. 2019.8747308

8. Hatta, T., Sawahara, M., Chiba, K.: Many-objective multidisciplinary evolutionary design for hybrid-wing-body-type flyback booster on an entirely automated system. In: Proceedings on International Conference on Evolutionary and Deterministic Methods for Design, Optimization, and Control with Applications to Industrial and Societal Problems 2019. ECCOMAS (2019)

9. Jones, D.R., Schonlau, M., Welch, W.J.: Efficient global optimization of expensive black-box functions. J. Global Optim. 13(4), 455-492 (1998)

10. Keane, A.J.: Wing optimization using design of experiment, response surface, and data fusion methods. J. Aircr. 40(4), 741-750 (2003)

11. Kolychev, A.V., Kernozhitskii, V.A., Chernyshov, M.V.: Thermionic methods of cooling for thermostressed elements of advanced reusable launch vehicles. Russ. Aeronaut. 62(4), 669-674 (2019). https://doi.org/10.3103/S1068799819040184

12. Sacha, D., Stoffel, A., Stoffel, F., Kwon, B.C., Ellis, G., Keim, D.A.: Knowledge generation model for visual analytics. IEEE Trans. Vis. Comput. Graph. 20(12), 1604-1613 (2014)

13. Shneiderman, B.: Extreme visualization: squeezing a billion records into a million pixels. In: Proceedings of the 2008 ACM SIGMOD International Conference on Management of Data, pp. 3-12 (2008). https://doi.org/10.1145/1376616.1376618

14. Simplício, P., Marcos, A., Bennani, S.: Reusable launchers: development of a coupled flight mechanics, guidance, and control benchmark. J. Spacecr. Rockets 56, 74-89 (2019). https://doi.org/10.2514/1.A34429

15. Sumimoto, T., Chiba, K., Kanazaki, M., Fujikawa, T., Yonemoto, K., Hamada, N.: Evolutionary multidisciplinary design optimization of blended-wing-body-type flyback booster. In: AIAA Paper 2019-0703 on the 57th AIAA Aerospace Science Meeting. AIAA (2019) 
16. Zhou, H., Wang, X., Cui, N.: Glide guidance for reusable launch vehicles using analytical dynamics. Aerosp. Sci. Technol. 98, 1-2 (2020). https://doi.org/10.1016/j. ast.2019.105678

17. Zitzler, E., Laumanns, M., Thiele, L.: SPEA2: improving the strength Pareto evolutionary algorithm. TIK-Report No. 103, Computer Engineering and Communication Networks Lab., ETH Zurich (2001) 